\title{
Asymptotic Analysis of a Deterministic Control System via Euler's Equation Approach
}

\author{
Gladys Denisse Salgado Suárez ${ }^{1}$, Hugo Cruz-Suárez ${ }^{1}$ \& José Dionicio Zacarías Flores ${ }^{1}$ \\ ${ }^{1}$ Facultad de Ciencias Físico Matemáticas, Benemérita Universidad Autónoma de Puebla, Puebla, México \\ Correspondence: Gladys Denisse Salgado Suárez, Facultad de Ciencias Físico Matemáticas, Benemérita Universidad \\ Autónoma de Puebla, Avenida San Claudio y 18 Sur, Colonia San Manuel, Ciudad Universitaria, C.P. 72570, Puebla, \\ México. E-mail: gladys008@ hotmail.com
}

Received: December 2, 2017 Accepted: December 22, 2017 Online Published: January 18, 2018

doi:10.5539/jmr.v10n1p115 URL: https://doi.org/10.5539/jmr.v10n1p115

\begin{abstract}
This paper provides necessary conditions in order to guarantee the existence of an unique equilibrium point in a deterministic control system. Furthermore, under additional conditions, it is proved the convergence of the optimal control sequence to this equilibrium point. The methodology to obtain these statements is based on the Euler's equation approach. A consumption-investment problem is presented with the objective to illustrate the results exposed.
\end{abstract}

Keywords: Deterministic control systems, Dynamic Programming, equilibrium point, Euler's equation, stability

\section{Introduction}

In this document a Deterministic Control System (DCS) is considered. A DCS is used to modelling dynamic systems, which are observed in a discrete time by a controller with the purpose that the system performs effectively with respect to certain optimality criteria (objective function). In this way a sequence of strategies to operate the dynamic system is obtained, this sequence is denominated a control policy. The optimal control problems consist in determining an optimal policy, i.e. a policy that minimizes the objective function. These classes of problems are included in the theory of Markov Decision Processes (Hernández-Lerma \& Jean, 1996; Hinderer, Rieder \& Stieglitz, 2017). In this context, a problem of interest is analysing the asymptotic behaviour of the optimal trajectory of the system, this problem is addressed in this document.

The convergence of the optimal trajectory of the DCS becomes of interest, in this work the necessary conditions for the convergence to its respective equilibrium point are provided. Important investigations base their results on the existence and characterization of the equilibrium point of the deterministic system associated with the stochastic system being studied (Judd \& Guu, 1993). The work is motivated from studying the economic growth model as it is exposed in De la Fuente (2000), where the stability of the system is guaranteed. In this document, we propose conditions for the stability of more general systems. The methodology that follows is to prove that the optimal trajectory is monotonous and bounded, thus demonstrating that it is convergent, later, the equilibrium point is characterized by the Euler's equation. Finally, it is proved that the convergence to the equilibrium point holds.

This paper is organized as follows. In Section 2, the deterministic system of control, main concepts and results in the theory of DCS are presented. In section 3 an essential result for the main objective of this work is developed. Finally, in Section 4, some concluding remarks are provided.

Notation: In the following sections, the derivative of a multi-variable function $M$ with respect to a variable $y$, it will be denoted by $M_{y}$, the gradient of the function $M$ is denoted by $D M(x, y)$ and the derivative of a function of a variable $h$ will be denoted by $h^{\prime}$.

\section{Materials and Methods}

A deterministic control model is conformed by the following components:

$$
(X, A,\{A(x) \mid x \in X\}, F, r),
$$

where $X=[0, \infty)$, is called the state space, $A=[0, \infty)$ is denominated the action space. $\{A(x) \mid x \in X\}$ is a family of measurable non-empty subsets $A(x)$ of $A$, here $A(x)$ denotes the set of feasible actions when the system is in state $x \in X$. $F: \mathbb{K} \rightarrow X$ is a known function with $\mathbb{K}=\{(x, a) \mid x \in X, a \in A(x)\}, r: \mathbb{K} \rightarrow \mathbb{R}$, is a known function called a one-step reward. 
The interpretation of the model is as follows: at each time $t \in\{0,1,2, \ldots\}$, we observe the system state $x_{t}=x \in X$, we apply an action $a_{t}=a \in A(x)$, and as a result we receive a reward $r(x, a)$ and the system is transferred to a new state $x_{t+1}$, determined by

$$
x_{t+1}=F\left(x_{t}, a_{t}\right),
$$

$t=0,1, \ldots$, with $x_{0}=x \in X$ initial state.

Let $\mathbb{F}=\{f: X \rightarrow A \mid f(x) \in A(x), x \in X\}$. A deterministic policy is a sequence $\pi=\left\{f_{t}: t=0,1, \ldots\right\}$ such that $f_{t} \in \mathbb{F}$, $t=0,1, \ldots$ The set of deterministic Markovian policies is denoted by $\mathbb{M}$. In particular, if $f_{t}=f \in \mathbb{F}$, for all $t \in\{0,1,2, \ldots\}$, $\pi=\{f, f, \ldots\}$ is called a stationary policy, and in this case the policy $\pi$ is only denoted by $f$.

Define for each $x \in X$ and $\pi \in \mathbb{M}$

$$
v(\pi, x):=\sum_{t=0}^{\infty} \alpha^{t} r\left(x_{t}, a_{t}\right)
$$

$v(\pi, x)$ is called the total discounted reward, where $\alpha \in(0,1)$ is the discount factor.

The objective of the control problem is to maximize $\pi \rightarrow v(\pi, x)$ on $\mathbb{M}$, for all $x \in X$, i.e., determining the optimal policy $\pi^{*}$ and optimal value function $V$, which are defined as follows.

Definition 1 It is defined for each $x \in X$

$$
V(x):=\sup _{\pi \in \mathbb{M}} v(\pi, x)
$$

$V$ is called the optimal value function or optimal value.

Definition 2 A policy $\pi^{*} \in \mathbb{M}$ is optimal, if

$$
v\left(\pi^{*}, x\right)=\sup _{\pi \in \mathbb{M}} v(\pi, x)
$$

Definition 3 The value iteration functions are defined as:

$$
V_{n}(x)=\max _{a \in A(x)}\left[r(x, a)+\alpha V_{n-1}(F(x, a))\right],
$$

for all $x \in X$ and $n=1,2, \ldots$, with $V_{0}(\cdot)=0$.

The following assumptions will be applied in the rest of the paper.

\section{Assumptions I}

a) $A(x)$ is a compact subset of $A$ for each $x \in X$.

b) $r$ and $F$ are continuous functions on $\mathbb{K}$.

c) There exist constants $c$ and $\beta$, with $1 \leq \beta<1 / \alpha$, and a function $w: X \rightarrow[1, \infty]$ such that for every state $x \in X$ :

i. $\sup _{a \in A(X)}|r(x, a)| \leq c w(x)$ and

ii. $\sup _{a \in A(X)}|w(F(x, a))| \leq \beta w(x)$.

\section{Assumptions II}

a) There exists a function $H: X \rightarrow X$ such that $\sup _{a \in A(X)} F(x, a) \leq H(x)$, for each $x \in X$. Furthermore, for the function $H$, there exists $x_{M} \in X$ such that $H\left(x_{M}\right)=x_{M}$.

b) There exists $\left(x^{\prime}, a^{\prime}\right) \in \mathbb{K}$ such that $\lim _{(x, a) \rightarrow\left(x^{\prime}, a^{\prime}\right)} F_{x}(x, a)=+\infty$.

c) $F_{x}(x, a) \rightarrow 0$ when $(x, a) \rightarrow+\infty$.

d) $V \in C^{2}$ and $V^{\prime \prime} \geq 0$.

e) $f \in C^{1}$ with $f^{\prime} \geq 0$.

Remark 1 In order to guarantee the differentiability of the optimal solution (see Assumption II d) and e)), you can consult the following references: De la Fuente (2000), Hernández-Lerma \& Jean (1996) and Cruz \& Montes-de-Oca (2008). Similarly, the concavity of the optimal value function and the monotonicity of the optimal policy can be consulted in Cruz, Montes-de-Oca \& Salem (2004) and Flores (2013), respectively.

The following theorem characterizes the optimal solution of the optimal control problem, via dynamic programming approach. 
Theorem 1 Suppose that Assumptions I hold, then:

a) The optimal value function $V$ is a solution for the following equation known as the Dynamic Programming Equation:

$$
V(x)=\max _{a \in A(x)}[r(x, a)+\alpha V(F(x, a))]
$$

$x \in X$.

b) There exists $f \in \mathbb{F}$ such that:

$$
V(x)=r(x, f(x))+\alpha V(F(x, f(x))),
$$

$x \in X$ and $f$ is optimal.

c) $V_{n}(x) \rightarrow V(x)$, as $n \rightarrow \infty$, for each $x \in X$ (see (1)).

Then, for Theorem $1 \mathrm{~b}$ ), there exists $f \in \mathbb{F}$ such that:

$$
x_{t+1}^{*}=F\left(x_{t}^{*}, f\left(x_{t}^{*}\right)\right):=g\left(x_{t}^{*}\right),
$$

$t=1,2, \ldots$, where $\left\{x_{t}^{*}\right\}$ represents the optimal trajectory of the control process. In this paper we are interested in studying the behaviour of (4) at its equilibrium point. According to Judd (1998), an equilibrium point $\bar{x} \in X$ associated with (4), satisfies the following identity:

$$
\bar{x}=F(\bar{x}, f(\bar{x}))=g(\bar{x}) .
$$

\section{Main Results}

This section presents the main result, which is to demonstrate that there exists an equilibrium point of (4) and the convergence of the optimal trajectory to this point.

Theorem 2 If $r$ and $F$ are concave functions and $r, F \in C^{2}(\mathbb{K})$ then the optimal policy, $f$, satisfies the following Euler equation:

$$
r_{a}(x, f(x))+\alpha \Delta(F(x, f(x)), f(F(x, f(x)))) F_{a}(x, f(x))=0, x \in(0, \infty)
$$

where $\Delta(x, a):=\left(r_{x}-r_{a} F_{x} / F_{a}\right)(x, a),(x, a) \in \mathbb{K}$.

Conversely, if $f \in \mathbb{F}$ is a policy which satisfies (6) for each $x \in(0, \infty)$ and

$$
\lim _{t \rightarrow \infty} \alpha^{t} \Delta\left(x_{t}, f\left(x_{t}\right)\right) x_{t}=0,
$$

then $f$ is an optimal policy.

Proof. Let $f$ be the optimal policy. Then by (2):

$$
V(x)=\max _{a \in A(x)}\{r(x, a)+\alpha V(F(x, a))\},
$$

in consequence the first-order condition is given by

$$
r_{a}(x, f(x))+\alpha V^{\prime}(F(x, f(x))) F_{a}(x, f(x))=0 .
$$

On the other hand, by (3):

$$
V(x)=r(x, f(x))+\alpha V(F(x, f(x)))
$$

then

$$
V^{\prime}(x)=r_{x}(x, f(x))+r_{a}(x, f(x)) f^{\prime}(x)+\alpha V^{\prime}(F(x, f(x)))\left[F_{x}(x, f(x))+F_{a}(x, f(x)) f^{\prime}(x)\right],
$$

substituting (8) in (9), it is obtained that

$$
V^{\prime}(x)=r_{x}(x, f(x))+\alpha V^{\prime}(F(x, f(x))) F_{x}(x, f(x)) .
$$

In consequence, from (8) and (10) follows that:

$$
\begin{aligned}
V^{\prime}(x) & =r_{x}(x, f(x))-\alpha \frac{r_{a}(x, f(x))}{\alpha F_{a}(x, f(x))} F_{x}(x, f(x)) \\
& =\left[r_{x}-\frac{r_{a} F_{x}}{F_{a}}\right](x, f(x)) \\
& =\Delta(x, f(x)) .
\end{aligned}
$$

Finally, substituting (11) in (8), the results follows. 
Now, will prove the converse. Let $f$ be a function that satisfies (6) and (7) and consider $x \in(0, \infty)$ fixed. Let $\hat{f} \in \mathbb{F}$ be another function and for $t=0,1, \ldots$, the trajectories of the policies $f$ and $\hat{f}$ be denoted by $x_{t}$ and $\hat{x}_{t}$, respectively. Similarly, $a_{t}=f\left(x_{t}\right)$ and $\hat{a}_{t}=\hat{f}\left(\hat{x}_{t}\right)$ denote their respective actions, where $x_{0}=\hat{x}_{0}=x$ for both.

Since $r$ is concave and $r \in C^{2}$, applying Theorem 2.17 in De La Fuente (2000), p.258, it is obtained that

$$
\begin{aligned}
\sum_{t=0}^{T-1} \alpha^{t}\left[r\left(x_{t}, a_{t}\right)-r\left(\hat{x}_{t}, \hat{a}_{t}\right)\right] & \geq \sum_{t=0}^{T-1} \alpha^{t}\left[\operatorname{Dr}\left(x_{t}, a_{t}\right)\left(x_{t}-\hat{x}_{t}, a_{t}-\hat{a}_{t}\right)\right] \\
& =\sum_{t=0}^{T-1} \alpha^{t}\left[r_{x}\left(x_{t}, a_{t}\right)\left(x_{t}-\hat{x}_{t}\right)+r_{a}\left(x_{t}, a_{t}\right)\left(a_{t}-\hat{a}_{t}\right)\right],
\end{aligned}
$$

for $T>1, T$ is a positive integer. Since $x_{t+1}=F\left(x_{t}, a_{t}\right)$ and $\hat{x}_{t+1}=F\left(\hat{x}_{t}, \hat{a}_{t}\right)$, then

$$
x_{t}-\hat{x}_{t}=F\left(x_{t-1}, a_{t-1}\right)-F\left(\hat{x}_{t-1}, \hat{a}_{t-1}\right) .
$$

Since $F$ is concave and $F \in C^{2}$, it results that

$$
F\left(x_{t-1}, a_{t-1}\right)-F\left(\hat{x}_{t-1}, \hat{a}_{t-1}\right) \geq F_{x}\left(x_{t-1}, a_{t-1}\right)\left(x_{t-1}-\hat{x}_{t-1}\right)+F_{a}\left(x_{t-1}, a_{t-1}\right)\left(a_{t-1}-\hat{a}_{t-1}\right),
$$

or equivalently

$$
\hat{a}_{t-1}-a_{t-1} \geq \frac{F_{x}\left(x_{t-1}, a_{t-1}\right)\left(x_{t-1}-\hat{x}_{t-1}\right)-\left(x_{t}-\hat{x}_{t}\right)}{F_{a}\left(x_{t-1}, a_{t-1}\right)},
$$

in consequence, it is obtained that

$$
\begin{aligned}
\sum_{t=0}^{T-1} \alpha^{t}\left[r_{x}\left(x_{t}, a_{t}\right)\left(x_{t}-\hat{x}_{t}\right)+r_{a}\left(x_{t}, a_{t}\right)\left(a_{t}-\hat{a}_{t}\right)\right] & \geq \sum_{t=0}^{T-1} \alpha^{t}\left[r_{x}\left(x_{t}, a_{t}\right)\left(x_{t}-\hat{x}_{t}\right)-r_{a}\left(x_{t}, a_{t}\right)\left(\frac{F_{x}\left(x_{t}, a_{t}\right)\left(x_{t}-\hat{x}_{t}\right)-\left(x_{t+1}-\hat{x}_{t+1}\right)}{F_{a}}\right)\right] \\
& \geq \sum_{t=1}^{T-1} \alpha^{t-1}\left(x_{t}-\hat{x}_{t}\right)\left[\alpha\left(r_{x}\left(x_{t}, a_{t}\right)-\frac{r_{a}\left(x_{t}, a_{t}\right) F_{x}\left(x_{t}\right)}{F_{a}\left(x_{t}, a_{t}\right)}\right)-\frac{r_{a}\left(x_{t-1}, a_{t-1}\right)}{F_{a}\left(x_{t-1}, a_{t-1}\right)}\right] \\
& -\alpha^{T-1} \frac{r_{a}\left(x_{T-1}, a_{T-1}\right)}{F_{a}\left(x_{T-1}, a_{T-1}\right)} x_{T} .
\end{aligned}
$$

Now, since $a_{t}=f\left(x_{t}\right)$ and $f$ satisfies (6), it follows that

$$
r_{a}\left(x_{T-1}, a_{T-1}\right)+\alpha \Delta\left(x_{T}, a_{T}\right) F_{a}\left(x_{T-1}, a_{T-1}\right)=0 .
$$

Now by (12), it is possible to conclude that

$$
\alpha^{T-1} \frac{r_{a}\left(x_{T-1}, a_{T-1}\right)}{F_{a}\left(x_{T-1}, a_{T-1}\right)} x_{T}=-\alpha^{T} x_{T} \Delta\left(x_{T}, a_{T}\right) .
$$

Similarly,

$$
\left(x_{t}-\hat{x}_{t}\right)\left[\alpha\left(r_{x}\left(x_{t}, a_{t}\right)-\frac{r_{a}\left(x_{t}, a_{t}\right) F_{x}\left(x_{t}, a_{t}\right)}{F_{a}\left(x_{t}, a_{t}\right)}\right)-\frac{r_{a}\left(x_{t-1}, a_{t-1}\right)}{F_{a}\left(x_{t-1}, a_{t-1}\right)}\right]=\left(x_{t}-\hat{x}_{t}\right)\left[\alpha \Delta\left(x_{t}, a_{t}\right)-\alpha \Delta\left(x_{t}, a_{t}\right)\right]=0 .
$$

Hence

$$
\sum_{t=0}^{T-1} \alpha^{t}\left[r\left(x_{t}, a_{t}\right)-r\left(\hat{x}_{t}, \hat{a}_{t}\right)\right] \geq \alpha^{T} x_{T} \Delta\left(x_{T}, a_{T}\right) .
$$

Then letting $T \rightarrow \infty$, by (7), it follows that

$$
\sum_{t=0}^{\infty} \alpha^{t} r\left(x_{t}, a_{t}\right) \geq \sum_{t=0}^{\infty} \alpha^{t} r\left(\hat{x}_{t}, \hat{a}_{t}\right) .
$$

Therefore $f$ is an optimal policy.

Theorem 3 Suppose that $r$ and $F$ are strictly concave functions and the Assumptions I and II hold. Then there exists an unique equilibrium point $\bar{x} \in X$ of $g$.

Proof. Firstly, observe that the equilibrium point $\bar{x}$, satisfies the following system equations:

$$
\begin{gathered}
\bar{x}=F(\bar{x}, f(\bar{x})) \\
r_{a}(\bar{x}, f(\bar{x}))+\alpha \Delta(F(\bar{x}, f(\bar{x})), f(F(\bar{x}, f(\bar{x})))) F_{a}(\bar{x}, f(\bar{x}))=0 .
\end{gathered}
$$

Equivalently

$$
r_{a}(\bar{x}, f(\bar{x}))+\alpha \Delta(\bar{x}, f(\bar{x})) F_{a}(\bar{x}, f(\bar{x}))=0 .
$$


Now, it will probed that (15) has an unique solution. Then consider the following function

$$
W(x):=r_{a}(x, f(x))+\alpha \Delta(x, f(x)) F_{a}(x, f(x)), x \in X .
$$

In particular, observe that $W(\bar{x})=0$.

Now, since $r$ and $F$ are strictly concave, it follows that $F_{a}$ and $r_{a}$ are non-negatives and strictly decreasing, as well as by Assumption II e) $f$ is increasing and due to Assumption II d) $V$ is strictly concave, therefore, the function $W$ defined in (16) is strictly decreasing. Substituting $\Delta$ and rewriting $W$, it is obtained that:

$$
W(x)=r_{a}(x, f(x))\left(1-\alpha F_{x}(x, f(x))\right)+r_{x} F_{a}(x, f(x)) .
$$

Then consider the following facts:

1. By Assumption II b), we can guarantee that there exists $z \in X$ such that $F_{x}(x, f(x)) \rightarrow+\infty$ as $x \rightarrow z$.

2. By Assumption II c) and the previous point, we can guarantee, that equation $F_{x}(x, f(x))-1 / \alpha=0$, has an unique solution, this solution will be denoted by $y$.

On the one hand, since $f$ is an increasing function (Assumption II e)) and $F$ is a strictly concave function, the function $1-\alpha F_{x}(x, f(x))$ is strictly increasing on $X$. In addition, it is satisfied that $1-\alpha F_{x}(y, f(y))=0$ by point 2 and $r_{x}(y, f(y)) F_{a}(y, f(y)) \geq 0$, therefore

$$
W(y)=r_{a}(y, f(y))\left(1-\alpha F_{x}(y, f(y))\right)+r_{x} F_{a}(y, f(y)) \geq 0 .
$$

Now by point 1 , there exists $z \in X$ such that $F_{x}(x, f(x)) \rightarrow+\infty$, as $x \rightarrow z$ then $1-\alpha F_{x}(x, f(x)) \rightarrow-\infty$ as $x \rightarrow z$, therefore

$$
W(z)=r_{a}(z, f(z))\left(1-\alpha F_{x}(z, f(z))\right)+r_{x} F_{a}(z, f(z)) \leq 0 .
$$

In conclusion, we found two elements $y$ and $z$ such that $W(y) \geq 0$ and $W(z) \leq 0$. Since $W$ is an strictly decreasing and continuous function, $W$ has an unique point $\bar{x} \in X$ such that $W(\bar{x})=0$, as a consequence of the intermediate value theorem. In conclusion, we find an unique equilibrium point of $g$, which is $\bar{x}$.

Remark 2 Observe that under Assumption II e), it holds the following statements:

a. If $F$ is an increasing function on $\mathbb{K}$ then the function $g$ defined in (4) is an increasing function.

b. If $F$ is a continuous function on $\mathbb{K}$ then $g$ is a continuous function on $X$.

Proposition 1 The optimal trajectory $\left\{x_{t}^{*}\right\}$ defined recursively by $x_{t+1}=g\left(x_{t}^{*}\right)$ with $x_{0} \in X$ fixed, is monotonous.

Proof. Since $V$ is strictly concave (Assumption II d)), then for $x^{\prime \prime}, x^{\prime} \in X$ :

$$
x^{\prime \prime}>x^{\prime} \text { implies that } V^{\prime}\left(x^{\prime \prime}\right)<V^{\prime}\left(x^{\prime}\right) \text {. }
$$

Consider two successive states of the optimal trajectory $x_{t}^{*}$ and $x_{t+1}^{*}$, where $x_{t+1}^{*}=g\left(x_{t}^{*}\right)$. Then, $x_{t}^{*}-x_{t+1}^{*}$ and $V^{\prime}\left(x_{t}^{*}\right)-V^{\prime}\left(x_{t+1}^{*}\right)$ will have opposite signs, that is

$$
\left(x_{t}^{*}-x_{t+1}^{*}\right)\left(V^{\prime}\left(x_{t}^{*}\right)-V^{\prime}\left(x_{t+1}^{*}\right)\right) \leq 0,
$$

in addition, by equations (11) and (8), it is obtained that

$$
\begin{gathered}
V^{\prime}\left(x_{t}^{*}\right)=\Delta\left(x_{t}^{*}, f\left(x_{t}^{*}\right)\right), \\
V^{\prime}\left(x_{t+1}^{*}\right)=-\left(r_{a} / \alpha F_{a}\right)\left(x_{t}^{*}, f\left(x_{t}^{*}\right)\right),
\end{gathered}
$$

then

$$
\left(x_{t}^{*}-x_{t+1}^{*}\right)\left[\Delta\left(x_{t}^{*}, f\left(x_{t}^{*}\right)\right)+\left(r_{a} / \alpha F_{a}\right)\left(x_{t}^{*}, f\left(x_{t}^{*}\right)\right)\right] \leq 0,
$$

multiplying by $\alpha F_{a}\left(x_{t}^{*}, f\left(x_{t}^{*}\right)\right)>0$ and rearranging, it is obtained that

$$
\left(x_{t}^{*}-x_{t+1}^{*}\right)\left[r_{a}\left(x_{t}^{*}, f\left(x_{t}^{*}\right)\right)+\alpha \Delta\left(x_{t}^{*}, f\left(x_{t}^{*}\right)\right) F_{a}\left(x_{t}^{*}, f\left(x_{t}^{*}\right)\right)\right] \leq 0 .
$$

Note that, under (15)

$$
W(\bar{x})=r_{a}(\bar{x}, f(\bar{x}))+\alpha \Delta(\bar{x}, f(\bar{x})) F_{a}(\bar{x}, f(\bar{x}))=0 .
$$

Then, since $W$ is decreasing and continuous, the following statements hold: 
- if $x_{0}>\bar{x}$ then since $g$ is increasing (Remark 2.a), $x_{t}^{*}>\bar{x}$. In consequence, $W\left(x_{t}^{*}\right)<W(\bar{x})=0$ and (17) implies that $x_{t}^{*}<x_{t+1}^{*}$, that is $\left\{x_{t}^{*}\right\}$ is a monotonous increasing sequence.

- Analogously, if $x_{0}<\bar{x}$ then $\left\{x_{t}^{*}\right\}$ is a monotonous decreasing sequence.

- if $x_{0}=\bar{x}$, then $x_{t}^{*}=x_{t+1}^{*}=\bar{x}$.

Remark 3 Note that if $x_{0}<\bar{x}$, by the proof of Proposition $1,\left\{x_{t}^{*}\right\}$ increases and if the Assumption II a) holds, then $\bar{x}<x_{M}$, in consequence $x_{0} \leq x_{t}^{*} \leq x_{M}$. Otherwise, $x_{0}>\bar{x}$, then $0 \leq x_{t}^{*} \leq x_{0}$. In this way, the compact in which $f_{n}$ converge to $f$ can be defined as $S:=\left[0, \max \left\{x_{M}, x_{0}\right\}\right]$.

Theorem 4 The optimal trajectory $\left\{x_{t}^{*}\right\}$ converge monotonously into $S \subseteq X$ to equilibrium point $\bar{x}$ for any initial value $x_{0} \in X$.

Proof. Since $\left\{x_{t}^{*}\right\}$ is a monotonous and bounded sequence (see Proposition 1 and Remark 3, respectively), then it converges. Let $x^{*}$ be the limit of sequence $\left\{x_{t}^{*}\right\}$. The, for the continuity of the function $g$ (Remark 2.b), $x^{*}$ must be a fixed point, because

$$
x^{*}=\lim _{t \rightarrow \infty} x_{t+1}^{*}=\lim _{t \rightarrow \infty} g\left(x_{t}^{*}\right)=g\left(\lim _{t \rightarrow \infty} x_{t}^{*}\right)=g\left(x^{*}\right)
$$

Then $x^{*}$ is an equilibrium point, but as $\bar{x}$ is the only equilibrium point, $x_{t}^{*} \rightarrow \bar{x}$.

\section{Example: Logarithmic Utility}

Consider an economy in which at each discrete time $t, t=0,1, \ldots$, there are $L_{t}$ consumers (population or labor), with consumption $a_{t}$ per individual, whose growth is governed by the following difference equation:

$$
L_{t+1}=L_{t} \eta
$$

it is assumed that initially the number of consumers, $L_{0}$, is known. In this case $\eta>0$ fixed represents an exogenous shock that affects the consumer population. In general, this shock is considered as a random variable, in this context $\eta$ represents the expected value (see Vitoriano, Werra, \& Parlier, 2016). The production function for the economy is given by

$$
Y_{t}=G\left(K_{t}, L_{t}\right)
$$

with $K_{0}$ known, i.e. the production $Y_{t}$ is a function of capital, $K_{t}$, and labor, $L_{t}$, where the production function, $G$, is a homogeneous function of degree one, i.e. $G(\lambda x, \lambda y)=\lambda G(x, y)$. The output must be split between consumptions $C_{t}=a_{t} L_{t}$ and the gross investment $I_{t}$, i.e.

$$
C_{t}+I_{t}=Y_{t} .
$$

Let $\delta \in(0,1)$ be the depreciation rate of capital. Then the evolution equation for capital is given by:

$$
K_{t+1}=(1-\delta) K_{t}+I_{t} .
$$

Substituting (20) in (19), it is obtained that

$$
C_{t}-(1-\delta) K_{t}+K_{t+1}=Y_{t}
$$

In the usual way, all variables can be normalized into per capital terms, namely, $y_{t}:=Y_{t} / L_{t}$ and $x_{t}:=K_{t} / L_{t}$. Then (21) can be expressed in the following way:

$$
a_{t}-(1-\delta) x_{t}+K_{t+1} / L_{t}=G\left(x_{t}, 1\right) .
$$

Now, using (18) in the previous relation, it yields that

$$
x_{t+1}=\xi\left(G\left(x_{t}, 1\right)+(1-\delta) x_{t}-a_{t}\right),
$$

$t=0,1,2, \ldots$, where $\xi:=(\eta)^{-1}$. Define $h(x):=G(x, 1)+(1-\delta) x, x \in X:=[0, \infty), h$ henceforth to be identified as the production function.

In particular, consider that $h$ is a power function, i.e. $h(x)=x^{\gamma}$, with $\gamma \in(0,1)$. Then, the transition law of the system is given by

$$
x_{t+1}=\xi\left(x_{t}^{\gamma}-a_{t}\right)
$$


$t=0,1,2, \ldots$, and $x_{0}=x \in X:=[0, \infty)$ is the initial capital. The admissible actions is given by $A(x)=\left[0, x^{\gamma}\right]$. The reward function is given by a consumption utility, $r(x, a)=U(a)=\ln a$, if $x \in(0, \infty), a \in\left(0, x^{\gamma}\right]$, and $r(0,0)=U(0)=\infty$. Furthermore, assume that $0<\alpha \gamma<1$.

Lemma 1 The logarithmic utility example satisfies Assumption I.

Proof. Firstly, observe that $r(x, a)=\ln (a)$ if $a \in\left(0, x^{\gamma}\right]$, and $r(x, 0)=\infty, x \in(0, \infty), F(x, a)=\frac{1}{\eta}\left(x^{\gamma}-a\right),(x, a) \in \mathbb{K}$. In consequence, Assumption I a) and Assumption I b) hold. Then it just necessary to prove Assumption I c), for this case consider $w(x)=\ln (x / \eta), x \in[0, \infty)$ and $c=1=\beta$.

Lemma 2 The logarithmic utility example satisfies Assumptions II.

Proof. a) Let $H(x)=x^{\gamma} / \eta$, be a continuous function mapping defined on the compact and convex set $X=[0, \infty)$. Then by Theorem 3.2 in De la Fuente (2000) p. 221, $H$ has a fixed point in $X$, this is indeed $x_{M}=\eta^{\frac{1}{\gamma-1}}$, in consequence $H\left(\eta^{\frac{1}{\gamma-1}}\right)=\eta^{\frac{1}{\gamma-1}}$. In this way, $F(x, a)=\frac{1}{\eta}\left(x^{\gamma}-a\right) \leq x^{\gamma}=H(x)$ for each $a \in A(x), x \in[0, \infty)$.

b) $F_{x}(x, a)=\frac{1}{\eta} \gamma x^{\gamma-1}$ and as $(x, a) \rightarrow(0,0), F_{x}(x, a) \rightarrow \infty$ because $\gamma \in(0,1)$.

c) When $(x, a) \rightarrow \infty, \frac{1}{\eta} \gamma x^{\gamma-1} \rightarrow 0$.

d) Firstly, observe that $V \in C^{2}$, due to $r$ and $F \in C^{2}$ (see Remark 1 and Cruz-Suárez (2008)). Furthermore, the second derivative of the value function si strictly positive, since $r$ and $F$ are strictly concave and increasing functions and the multifunction, $x \mapsto A(x)$, is increasing and convex function.

e) Since $r$ and $F \in C^{2}$, by Remark 1, $f \in C^{1}$ and due to the multifunction $x \rightarrow A(x)$ is increasing and convex, by Remark $3, f$ is increasing.

Lemma 3 The equilibrium point is $\bar{x}=\left(\frac{\eta}{\alpha \gamma}\right)^{\frac{1}{\gamma-1}}$.

Proof. The equilibrium point is characterized by equations (5) and (15). Then substituting the respective values in (5) and (15), it results that

$$
\left\{\begin{array}{c}
\frac{1}{f(\bar{x})}\left(1-\frac{\alpha \gamma \bar{x}^{\gamma-1}}{\eta}\right)=0, \\
\frac{1}{\eta}\left(\bar{x}^{\gamma}-f(\bar{x})\right)=\bar{x} .
\end{array}\right.
$$

Solving the previous system for $\bar{x}$ and $f(\bar{x})$, it is obtained that:

$$
\begin{gathered}
\bar{x}=\left(\frac{\eta}{\alpha \gamma}\right)^{\frac{1}{\gamma-1}} \\
f(\bar{x})=\left(\frac{\eta}{\alpha \gamma}\right)^{\frac{\gamma}{\gamma-1}}\left(1-\frac{\alpha \gamma}{\eta}\right) .
\end{gathered}
$$

In conclusion, for this example, the optimal trajectory converges to $\bar{x}=\left(\frac{\eta}{\alpha \gamma}\right)^{\frac{1}{\gamma-1}}$.

The Figure 1 represents the first ten realization of the optimal trajectory. 


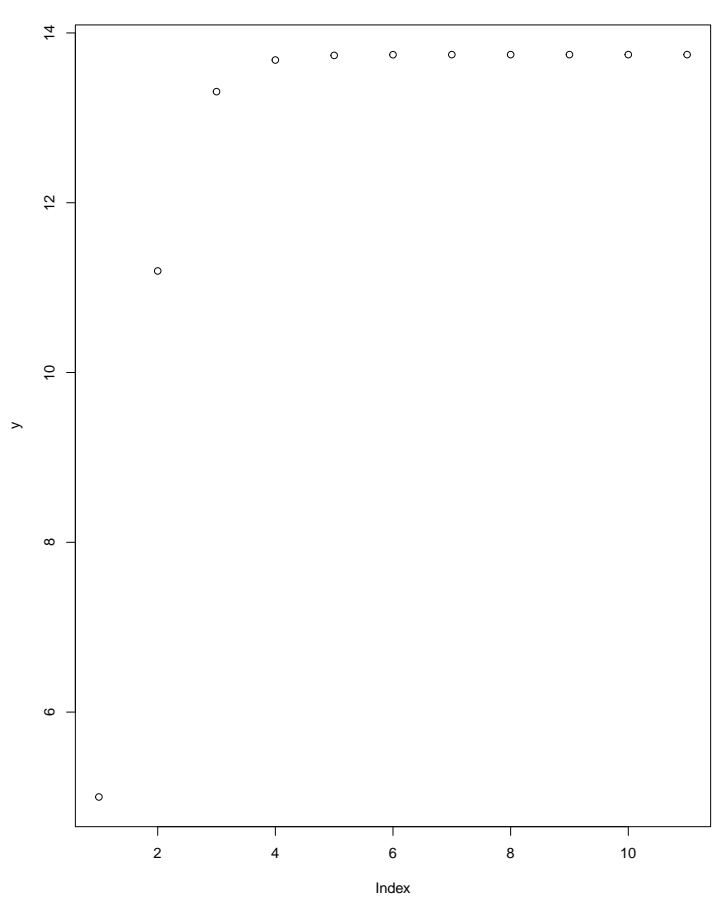

Figure 1. Optimal Trajectory

\section{Conclusions}

In this work conditions on the model which guarantee the convergence of the optimal trajectory of the DCS are established. In specific, a compact subset $S$ of the real numbers is characterized (see Remark 3). Then, it is proved that the optimal trajectory is concentrated in the set S. Finally, it is shown that the optimal trajectory is converging to the equilibrium point of the DCS. Future works in this direction, consists in studying a stochastic control problem induced by the DCS (Cruz $\&$ Montes-de-Oca, 2006), and in this case, we are interested in guaranteeing the convergence of the stochastic optimal trajectory.

\section{References}

Cruz-Suárez, H., \& Montes-de-Oca, R. (2006). Discounted Markov control processes induced by deterministic systems. Kybernetika, 42(6), 647-664.

Cruz-Suárez, H., \& Montes-de-Oca, R. (2008). An envelope theorem and some applications to discounted Markov decision processes. Mathematical Methods of Operations Research, 67(2), 299-321.

Cruz-Suárez, D., Montes-de-Oca, R., \& Salem, F. (2004). Conditions for the uniqueness of optimal policies of discounted Markov decision processes. Mathematical Methods of Operations Research, 60(3), 415-436.

De La Fuente, A. (2000). Mathematical Methods and Models for Economists. Cambridge University Press.

Flores-Hernández, R. M. (2013). Monotone optimal policies in discounted Markov decision processes with transition probabilities independent of the current state: existence and approximation. Kybernetika, 49(5), 705-719.

Hernández-Lerma, O, \& Jean B. L. (1996). Discrete-time Markov control processes: basic optimality criteria., 30. Springer Science and Business Media.

Hinderer, K., Rieder, U., \& Stieglitz, M. (2017). Dynamic Optimization. Springer International Publishing AG.

Judd, K. L. (1998). Numerical methods in economics. MIT press.

Judd, K. L., \& Guu, S. M. (1993). Perturbation solution methods for economic growth models. In Economic and Financial Modeling with Mathematica, Springer New York. 80-103.

Vitoriano, B. De Werra, D., \& Parlier, G. H. (2016) Operations Research and Enterprise Systems, in 5th International Conference on Operations Research and Enterprise Systems, Rome, Italy. 


\section{Copyrights}

Copyright for this article is retained by the author(s), with first publication rights granted to the journal.

This is an open-access article distributed under the terms and conditions of the Creative Commons Attribution license (http://creativecommons.org/licenses/by/4.0/). 